\title{
PROBE VEHICLE TRACK-MATCHING ALGORITHM BASED ON SPATIAL SEMANTIC FEATURES
}

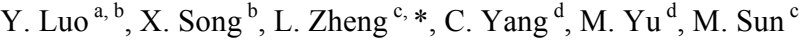 \\ ${ }^{a}$ School of Resource and Environmental Sciences, Wuhan University, China - yuejunl@kotei-info.com \\ ${ }^{\mathrm{b}}$ Wuhan KOTEI Infomatics Co., Ltd. China - (yuejunl, xiangbos)@kotei-info.com \\ ${ }^{\mathrm{c}}$ School of Geodesy and Geomatics, Wuhan University, China - lzheng@sgg.whu.edu.cn, mengsun_2015@126.com \\ ${ }^{\mathrm{d}}$ Department of Geography and GeoInformation Sciences, College of Science, George Mason University, Fairfax, VA 22030, \\ USA - (cyang3, myu7)@gmu.edu
}

KEY WORDS: Spatial Semantic Features, Probe Vehicle Path, Spatial Data Mining, Global Matching Model, Track

\begin{abstract}
:
The matching of GPS received locations to roads is challenging. Traditional matching method is based on the position of the GPS receiver, the vehicle position and vehicle behavior near the receiving time. However, for probe vehicle trajectories, the sampling interval is too sparse and there is a poor correlation between adjacent sampling points, so it cannot partition the GPS noise through the historical positions. For the data mining of probe vehicle tracks based on spatial semantics, the matching is learned from the traditional electronic navigation map matching, and it is proposed that the probe vehicle track matching algorithm is based on spatial semantic features. Experimental results show that the proposed global-path matching method gets a good matching results, and restores the true path through the probe vehicle track.
\end{abstract}

\section{INTRODUCTION}

With the rapid development of urbanization and society, vehicles have played a significant role in human life, and yet caused various problems to environment and economy. As a new-generation advanced navigation data acquisition mode, probe vehicle technology provides more possibilities to solve the above series of problems. Probe vehicle, called GPS probe car, is used in Intelligent Transport System (ITS) to obtain the urban road traffic state as the effective way in recent years (Geng, 2013).

Probe vehicle is an advanced road traffic information collection technology in ITS field in the current internation (Hao, 2012). The data range of probe vehicle technology can be distributed in all areas and collect data for 24 hours. Using wireless real-time transmission, centralized processing can greatly improve the efficiency of information acquisition. At the same time, the use of existing resources of GPS and communication network make the acquisition equipment maintenance and installation costs lower (Zhu, 2009). Therefore, the probe vehicle technology is more economical and efficient method. Since point-by-point, nearest road matching often fails, researchers have developed methods that match several points at once. Kim and Kim (Krumm, 2008) look at a way to measure how much each GPS point belongs to any given road, taking into account its distance from the road, the shape of the road segment, and the continuity of the path. The measure is used in a fuzzy matching scheme with learned parameters to optimize performance. Brakatsoulas et al. (Brakatsoulas, et al., 2005) uses variations of the Fréchet distance to match the curve of the GPS trace to candidate paths in the road network. They tested their algorithms on 45 routes in Athens, Greece. Alt et al. (Alt, et al. 2003) give a generalization of the Fréchet for matching curves. Hidden Markov Models (HMM) solve this problem by explicitly modeling the connectivity of the roads and considering many different path hypotheses simultaneously.
To take advantage of probe vehicle technology in spatial data mining, it needs accurately reconstruct the real path that probe vehicle track through, and achieve the spatial registration of floating vehicle trajectory data and the real road network data. The probe vehicle trajectories data has some data characteristics such as large sample interval and position noise, which makes electronic map matching of the traditional method not suitable for the path matching of probe vehicle trajectories. Therefore, it is difficult to conduct the spatial data mining in probe vehicle trajectories. We will compare and contrast this work with ours subsequently after we explain the details of our algorithm.

\section{PROBLEMS IN TRADITIONAL PATH MATCHING ALGORITHM}

The traditional method of navigation map matching mainly calculate of current vehicle locations by GPS location information received, and then realize the matching of the vehicle in the road net using the point line distance or curve distance. Due to the existence of GPS noise error, the matching result is often modified through history track and vehicle motion information, in order to eliminate the influence of GPS noise in a certain degree (Geng, 2013; Su, 2001; Tang, 2008). Because the GPS sampling point interval is only $1 \mathrm{~s}$, the traditional navigation map matching algorithm is easier to obtain accurate trajectory curve as matching samples, and can realize line to line map matching based on the track curve. However, the probe vehicle trajectories usually upload a GPS data in the tens of seconds to a minute due to large sampling intervals. At the speed of $40 \mathrm{~km} /$ hour of probe vehicle, GPS frequency per minute has around a difference of more than 600 meters, where there may be multiple paths within this distance. Poor correlation between two points before and after determines that probe vehicle system cannot use the line to line map matching method. The raw input data consists of vehicle locations measured by GPS like most other map matching work in the paper. Each measured point consists of a time-stamped latitude/longitude pair. The roads are also represented in the

\footnotetext{
* Corresponding author
} 
conventional way. If it is still used the traditional matching method of navigation map, it may lead to the wrong path, and even the whole trajectory which match up with a passable path(as shown in Figure 1). Obviously, the traditional navigation map matching algorithm cannot be directly applied to the probe vehicle system (Wang, 2012).( Black is the map of the road network, the green line for probe vehicle trajectories, red for trajectory matching results).

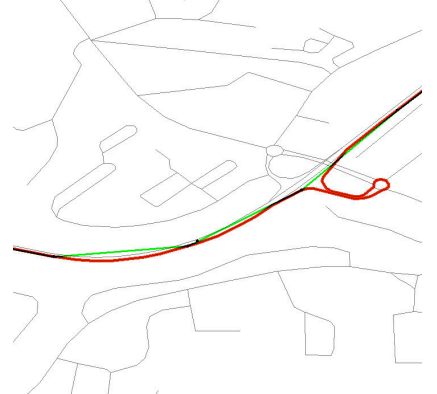

Figure 1 Probe vehicle and its true path and road network

\section{GLOBAL MATCHING MODEL BASED ON SPATIAL SEMANTIC FEATURE}

Matching problem model of traditional navigation map make a match only according to the position of the GPS receiver, the vehicle position and vehicle behavior near the time. Compared with the entire route, it is a local path matching. For probe vehicle trajectories, the sampling interval is too large and there is a poor correlation between adjacent sampling points, so it cannot make the GPS noise correct through the historical position. At the same time, GPS points of each sample are affected by the noise, so the path matching using local point alone is likely to match the wrong road, eventually it will lead to the whole trajectory matching to the wrong path. The literature (Sinn, 2001) proposed method of trajectory point fuzzy matching. To a certain extent, it can reduce the influence of noise, but still select in the local optimal path.

From the perspective of information fusion (Sotirls, 2005), For offline maps match like probe vehicle track matching (Pereiraf, 2009), it can also examine the position information of all the sampling points on the track. Between these different sampling points, there can be some behavior such as access, limited access time. Therefore, it can be considered the overall trajectory of probe vehicle, and make the optimal trajectory of global matching.

Based on the above analysis, it proposes the following float trajectory path matching method based on spatial semantic features.

1) Each track is regarded as a proof of information to restore the true path of trajectory.

2) Each trajectory point must be in the scope of the fuzzy matching in the road net.

3) According to a path way to explore, fuzzy matching results for all track points are connected in turn, then get all the possible global matching path.

4) According to the evaluation standards of some space semantic, searching a global optimal matching path in the global matching path.

Set all possible global matching path from the global matching

path model as $P=\left\{p_{t}, t=1,2, \cdots, l\right\}$, the path $p^{t}$ is made up of road sequence $\left\{l_{t i}, i=1,2, \cdots, t_{i m_{t}}\right\}$.

The length of the path $p_{t} L=\sum_{i=1}^{m_{t}} l_{t i}, l_{t_{i}}$ means the length

of the road $i$.

Road category changes of the road $p_{t} K=\sum_{i=1}^{m_{t}-1}\left(f\left(l_{i}, l_{i+1}\right)\right)$,

$f(x, y)=\left\{\begin{array}{l}1, x=y \\ 0, x \neq y\end{array}\right.$

Turn number of the path $p_{t} C=\sum_{i=1}^{m_{t}-1}\left(g\left(l_{i}, l_{i+1}\right)\right)$, where $g(x, y)=\left\{\begin{array}{l}1, \text { angle }<\text { threshhold } \\ 0, \text { others }\end{array}\right.$

At the same time, Set the path number of the road $p_{t}$ as

$M=m_{t}$, Track points the path $p_{t}$ passing by are $N_{t}$

In order to evaluate the advantages and disadvantages of a selected path, it is present a spatial semantic features $\Phi=\{L, K, C, M, N\}$ with \{length, road category changes, turn number, road numbers, track points $\}$ to describe the path. So the spatial semantic feature of the path $p_{t}$ is $\Phi_{t}=\left\{L_{t}, K_{t}, C_{t}, M_{t}, N_{t}\right\}$

\section{THE SPATIAL SEMANTIC FEATURE REDUCTION METHOD BASED ON PROBE VEHICLE TRAJECTORIES}

According to the global matching model based on spatial semantic feature and selected spatial semantic feature, it is proposed float trajectory path restoration methods based on spatial semantic features.

\subsection{Track point path searching}

With each float trajectory point as the center, matching all the way within a certain value $\left({ }^{r_{0}}\right)$. Set the road collection get by an fuzzy match as $L_{i}\left\{l_{i 1}, l_{i 2}, \cdots, l_{i m_{i}}\right\}, m_{i}$ is the road numbers matched by track points i. Searching a path among all links matching in two adjacent track points.

For example, while searching between track point i and track point $\mathrm{i}+1$, there will be $\left(l_{i j}, l_{i+1, k}\right)$ for any combination of $l_{i j}\left(j=1,2, \cdots, m_{i}\right)$ and $l_{i+1, k}\left(k=1,2, \cdots, m_{i+1}\right)$ where $\left(j \in\left\{1,2, \cdots, m_{i}\right\} ; k \in\left\{1,2, \cdots, m_{i+1}\right\}\right)$. Searching an accessible path $r_{i_{j k}}\left(l_{i j}, l_{i n_{1}}, \cdots, l_{i n_{i}}, l_{i+1, k}\right)$ in a certain threshold $S_{0}$ of the road number using the Dijstra algorithm, where $\left(n_{i} \geq 0\right)$. If the accessible path is not found in the threshold range, the combination will be abandoned. 


$$
\begin{aligned}
& \begin{array}{l}
\text { Finally, } \\
R_{i}=\left\{r_{i_{j k}}\left(l_{i j}, l_{i n_{1}}, \cdots, l_{i n_{i}}, l_{i+1, k}\right)\right\}_{\text {between }}^{\text {local track point } \mathrm{i} \text { and }} \\
\text { track } \quad \begin{array}{c}
\mathrm{i}+1, \\
\text { point }
\end{array}
\end{array} \\
& \left(j=1,2, \cdots, m_{i} ; k=1,2, \cdots, m_{i+1} ; n_{i} \geq 0\right)
\end{aligned}
$$

\subsection{Optimal path selection based on spatial semantic features}

For above all possible access route $R_{i}$ between adjacent track points, where $(i=1,2, \cdots, n)$, connecting into a full path by choosing connecting path with track points as much as possible.

$p_{t}$ generation algorithm flow chart is as shown in Figure 2 .

According to this algorithm, it can be obtained the set of all the full path, which is $P=\left\{p_{t}, t=1,2, \cdots, l\right\}$. In the full path of all, selecting the optimal a full path as the route of the trajectory by the formula, which is $p_{\text {opt }}=\min _{t=1,2, \cdots, l} \varphi\left(p_{t}\right) \varphi\left(p_{t}\right)_{\text {is }}$ the spatial semantic characteristic function of $p_{t}$

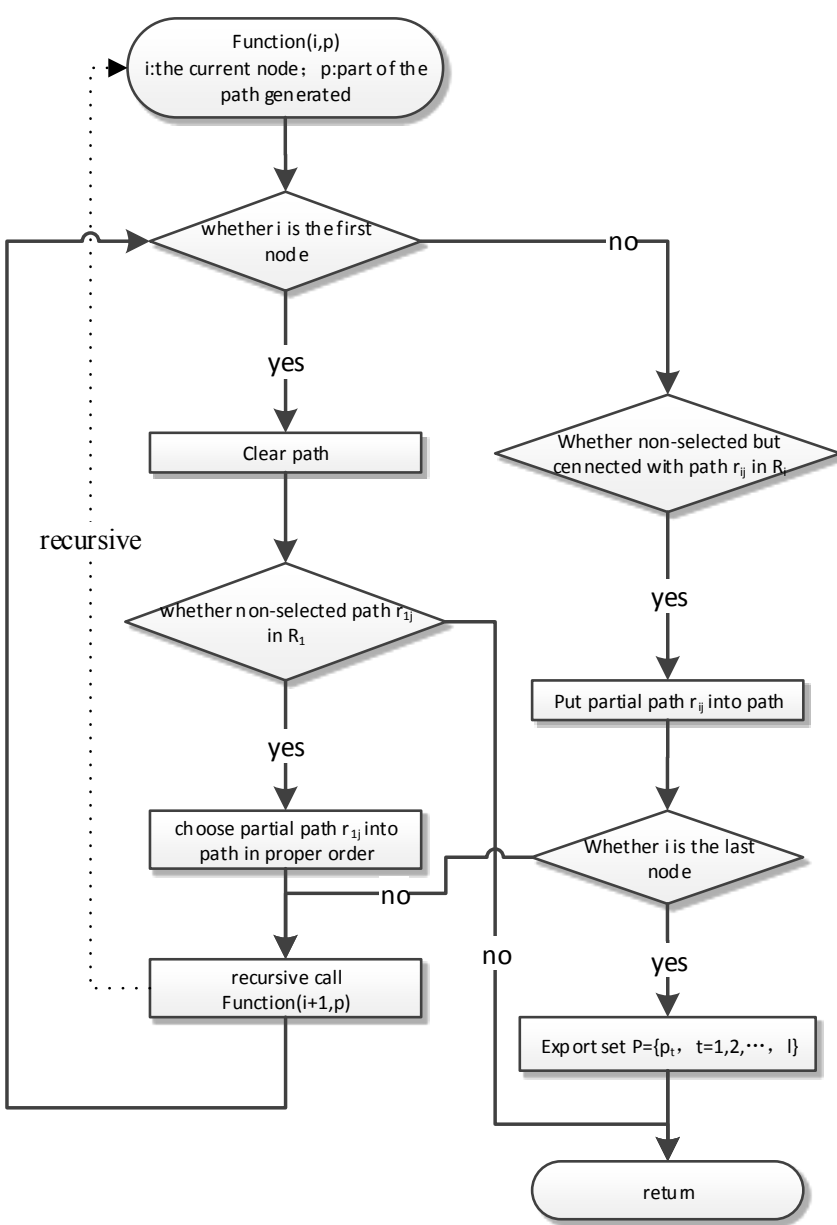

Figure 2 The flow chart of the global full path generation

\section{EXPERIMENTAL VERIFICATION}

By using the method in this paper, the path matching reduction experiment is completed by random sampling 300 vehicle acquisition track. In this experiment, the fuzzy matching threshold $\mathrm{r} 0$ of track points is 50 meters, the road number threshold $\mathrm{S} 0$ is 15 . As shown in the experimental results, there are 300 tracks matching complete route. Through visual inspection, there are 269 tracks which are restored ideally, accounting for $89.66 \%$. Figure $3 \sim$ Figure 5 respectively means practical trajectory matching results. (Black is the map of the road network, the green line for probe vehicle trajectories, red for trajectory matching results)

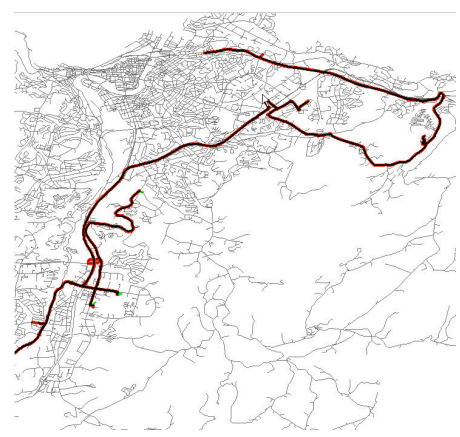

(a)

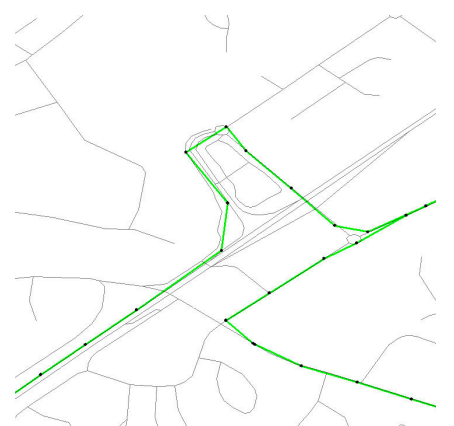

(b)

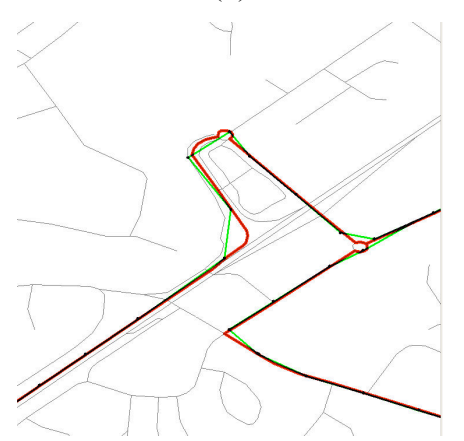

(c)

Figure 3 The overall view and a partial enlarged view by tracks 1 matching

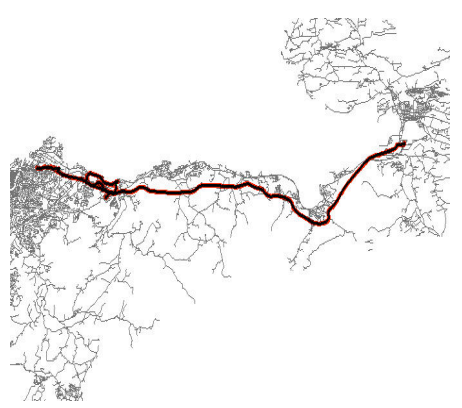

(a) 


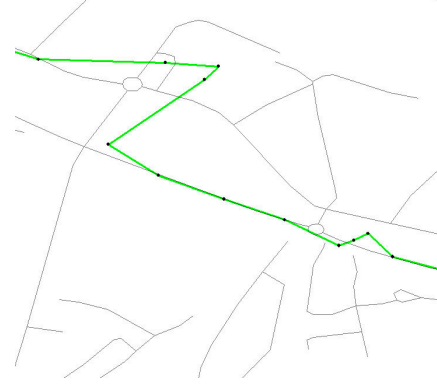

(b)

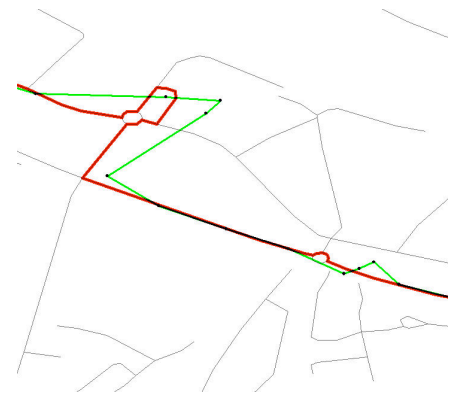

(c)

Figure 4 The overall view and a partial enlarged view by tracks 2 matching

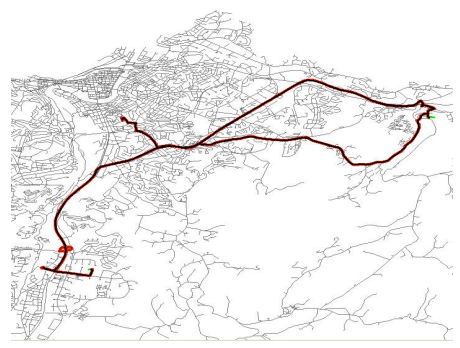

(a)

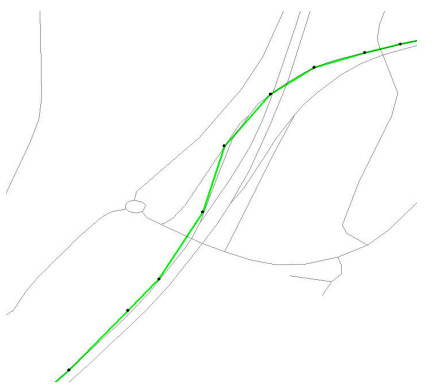

(b)

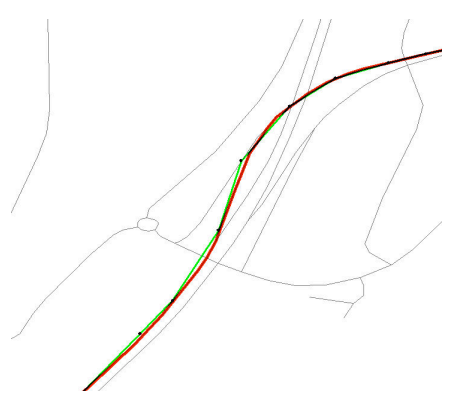

(c) view by tracks 5 matching

\section{CONCLUSION}

For the spatial semantic analysis problem in probe vehicle track data mining, this paper describes the main problem when the traditional navigation electronic map matching method is used for probe vehicle track map matching. And it puts forward the probe vehicle trajectories matching technology based on spatial semantic features. Through the actual data, the method presented in this paper is verified by experiment. Experiments show that matching method proposed in this paper gets a better match, and restores the real path float trajectory through.

Since the method proposed in this paper requires all possible paths of the probe vehicle trajectories to make the comparison operator, so as to select the optimal path. The computation and consumption of hardware resources is too large. Thus when the probe vehicle track contains more track points, the performance of the algorithm needs further optimization.

\section{ACKNOWLEDGMENT}

The authors would like to thank the anonymous reviewers and members of the editorial team for the comments and contributions. This work is supported by Digital Cartography and Land Information Application Engineering Key Laboratory Open Research Fund of National Administratoin of Surveying, Mapping and Geoinformation and by 2014 Youth Science and Technology Chenguang Plan of Hubei Province (China) and by the National High Technology Research and Development Program of China (863 Program) (No. 2013AA12A202)

\section{REFERENCES}

Alt, H., et al., 2003. Matching Planar Maps. Journal of Algorithms, 49: pp. 262-283.

Brakatsoulas, S., et al., 2005. On Map-Matching Vehicle Tracking Data, in 31st International Conference on Very Large Databases (VLDB 2005).:Trondheim, Norway p. 853- 864.

Dai, X., Ferman, M., Roesser R P. 2003. A simulation evaluation of a real-time traffic information system using probe vehicles, Intelligent Transportation Systems, Proceedings. IEEE, pp:475-480.

Geng, X.,WANG, S., Ji, J., 2013. Fast Road -Matching Algorithm of Floating Car. Journal of Water Resources and Architectual Engineering, Vol11,No.1:122-125.

Gou, X., Zuo, X., Zhang, Y., 2013. Application of Digital Velocity Model in Urban Traffic Dynamics Analysis Based on probe-car. Science Technology and Engineering, Vol13,No.11: 3172-3177.

Hao, Y., Wu, G., Zhou, S., 2012. A Multi-vehicle Speed Fusion Algorithm Based on Probe Vehicle Data. Journal of Transport Information and Safety, Vol30, No.169: 56-61.

Krumm, J., 2008. A Markov Model for Driver Turn Prediction, in Society of Automotive Engineers (SAE) World Congress. Detroit, Michigan, USA.

Li, X., Meng, Q., 2002. The Applications of GPS Technology in the Real-Time Detection of City Traffic Condition To GPS. Journal of Ocean University of Qingdao:natural science edition, 32(3): 475-481.

Li, Q., Yin, J., He, F., 2009. A Coverage Rate Model of GPS probe-car for Road Networks. Geomatics and

Figure 5 The overall view and a partial enlarged 
Information Science of Wuhan University, 34(6): 715718.

Pereir, F., Costa, H., 2009, An off-line map-matching algorithm for incomplete map databases. European Transportation Research Review, 1(3):107-124.

Schafer, R., Thiessenhusen, K., Wagner, P., 2002. A traffic information system by means of real-time floating-car data. ITS world congress.

Sinn, K., Jong, H., Adaptive Fuzzy-Network-Based C-Measure Map-Matching Algorithm for Car Navigation System. IEEE Transactions on Industrial Electronics, Vo1.48, No.2.

Sinn, K., Jong, K., 1999,Q-factor Map Matching Method Using Adaptive Fuzzy Network. IEEE International Fuzzy Systems Conference Proceedings. pp22-25.

Sotirls, B,. 2005, On Map-Matching Vehicle Tracking Data. Proceeding of the 31st VLDB Conference.

Su, J., Zhou, D., Yue, C,. 2001,Real-time Map-matching Algorithm in GPS Navigation. Acta Geodaetica et Cartographica Sinica,30(3):252-256.

Tang, J., Cao, K., 2008, An Adaptive Trajectory Curves Mapmatching Algorithm. Acta Geodaetica et Cartographica Sinica,37(3),pp:308-315.

Wang, M., Cheng, L., 2012, Real-time Map-matching Algorithm in GPS Navigation System for Vehicles. Acta Geodaetica et Cartographica Sinica,2 , Vol41,No.1: 133-138.

Zhang, C., 2007, Research on the Traffic Data Collection and Data Processing Theory and Method based on probecar. Shanghai:Tongji University.

Zhang, Z., Lin, X., Lin, S., 2011, Traffic Parameter Features in Traffic Incidents Based on Probe-car Data. Journal of Transport Information and Safety,29(3): 94-98.

Zhu, T., Guo, S., 2009,A Study on probe-car Based Information Processing Technology. Joumal of Image and Graphics.Vol14,No.7:1230-1237. 